\title{
Elastic and Piezoresistive Properties of Nickel Carbides from First-Principles
}

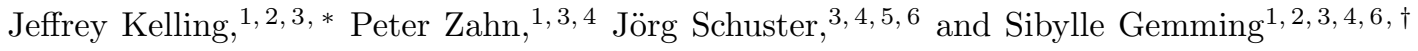 \\ ${ }^{1}$ Helmholtz-Zentrum Dresden - Rossendorf, Institute of Ion Beam Physics \\ and Materials Research, Bautzner Landstraße 400, 01328 Dresden, Germany \\ ${ }^{2}$ Institute of Physics, TU Chemnitz, 09107 Chemnitz, Germany \\ ${ }^{3}$ Helmholtz-Zentrum Dresden - Rossendorf, International Helmholtz Research School for \\ Nanoelectronic Networks (IHRS NanoNet), Bautzner Landstraße 400, 01328 Dresden, Germany \\ ${ }^{4}$ Dresden Center for Computational Materials Science (DCMS), TU Dresden, 01062 Dresden, Germany \\ ${ }^{5}$ Fraunhofer Institute for Electronic Nano Systems (ENAS), Technologie-Campus 3, 09126 Chemnitz, Germany \\ ${ }^{6}$ Center for Advancing Electronics Dresden (cfaed), TU Dresden, 01062 Dresden, Germany
}

\begin{abstract}
The nickel-carbon system has received increased attention over the past years due to the relevance of nickel as a catalyst for carbon nanotube and graphene growth, where Nickel carbide intermediates may be involved or carbide interface layers form in the end. Nickel-carbon composite thin films comprising $\mathrm{Ni}_{3} \mathrm{C}$ are especially interesting in mechanical sensing applications. Due to the metastability of nickel carbides, formation conditions and the coupling between mechanical and electrical properties are not yet well understood. Using first-principles electronic structure methods, we calculated the elastic properties of $\mathrm{Ni}_{3} \mathrm{C}, \mathrm{Ni}_{2} \mathrm{C}$ and $\mathrm{NiC}$, as well as changes in electronic properties under mechanical strain. We observe that the electronic density of states around the Fermi level does not change under the considered strains of up to $1 \%$, which correspond to stresses up to $3 \mathrm{GPa}$. Relative changes in conductivity of $\mathrm{Ni}_{3} \mathrm{C}$ range up to maximum values of about $10 \%$.
\end{abstract}

\section{INTRODUCTION}

Nickel-carbon compounds and composite thin films containing amorphous carbon are of high interest for various applications. Thin films have been investigated for their piezoresistive properties ${ }^{1}$ and as low friction solid lubricants 2 . The meta-stable $\mathrm{Ni}_{3} \mathrm{C}$ has been frequently observed in such films $3[5$ and was suggested to cause piezoresistive behaviour ${ }^{1}$. This carbide has been reported to be hard to distinguish from hcp-nickel, where a study ${ }^{\sqrt{5}}$ suggests that hcp-nickel is only stable in the presence of carbon and with some carbon content. A meta-study on this subject can be found in reference 4. A recent study confirmed, that $\mathrm{Ni}_{3} \mathrm{C}$ in such films only decomposes at temperatures well above $250{ }^{\circ} \mathrm{C}$.

The nickel-carbon system is also of interest for the catalytic production of carbon nanotubes (CNTs) and graphene. CNT growth was achieved both using nickel nanoparticles as a catalyst ${ }^{7 / 8}$ and on carbon-nickel nanocomposite thin films $s^{9}$. While studies suggest, that carbides do not form during CNT growth from $\mathrm{Ni}$ nanoparticles $10, \mathrm{Ni}_{3} \mathrm{C}$ has been observed in nanoparticles after CNT growth by plasma enhanced chemical vapor deposition was stopped 11 . A more recent study 12 confirmed, that $\mathrm{Ni} / \mathrm{Ni}_{3} \mathrm{C}$ core-shell structures can indeed be produced. In such a setup, the carbide could act as an advanced contact material for CNT junctions with properties similar to those demonstrated for $\mathrm{Mo}_{2} \mathrm{C} 13$. The advantage would be, that the $\mathrm{Ni}_{3} \mathrm{C}-\mathrm{CNT}$ unit can be grown bottom-up. $\mathrm{Ni}_{3} \mathrm{C}$ does also occur as a parasitic by-product of carbon nanofiber-growth on nickel foam 14 .

Graphen $e^{\sqrt{15}}$ and graphene-type interfacial layers ${ }^{16}$ can be produced by metal-induced crystallization and layer inversion as well as by epitaxial growth on transition metals, such as nicke $e^{17}$. In the latter case, one study 18 excluded the occurrence of crystalline $\mathrm{Ni}_{3} \mathrm{C}$ on a poly- crystalline Ni surface by XRD measurements. Others observed an interface layer between $\{111\}$-nickel and graphene with the stoichiometry $\mathrm{Ni}_{2} \mathrm{C}$ by Auger spectroscopy 19120 . In both cases, mechanical details, especially of carbide intermediates require further study.

The stability of a range of nickel-carbides has been investigated by density functional calculations 21 , yet, neglecting the influence of elastic deformations which we address here. The study confirmed that, without externally induced strains, $\mathrm{Ni}_{3} \mathrm{C}$ in space group 167 structure, figure 1|(c) is the least unstable carbide and suggests that $\mathrm{Ni}_{2} \mathrm{C}$ is most stable in orthorhombic structures of space groups 058 (Pnnm) and 060 (Pbcn), see figures 1) and (b).

All in all, especially the phase $\mathrm{Ni}_{3} \mathrm{C}$ has potential technical applications in heterojunctions consisting of nickel and carbon allotropes, including CNTs, acting as electrical circuit elements, for example piezoresistive sensors ${ }^{22}$. In these applications, the mechanical and piezoresistive properties of a potential carbide layer between nickel and the carbon structure can become relevant when the device is being strained during operation or when the layer is under constant epitaxial stress which may be caused the large surface tension of nickel 23 .

The present work focuses on investigating the elastic properties of the three nickel carbides $\mathrm{NiC}, \mathrm{Ni}_{2} \mathrm{C}$ and $\mathrm{Ni}_{3} \mathrm{C}$ in their most stable crystallographic structures. Ground state properties of the carbides are compared in section IIIA, the obtained elastic properties are discussed in section IIIB, For the experimentally most relevant carbide, $\mathrm{Ni}_{3} \mathrm{C}$, the influence of strain on the electronic transport properties is dicussed in section IIIC. 


\section{COMPUTATIONAL METHODS}

\section{A. Electronic Structure Calculations}

$\mathrm{NiC}$ was calculated in rocksalt (B1) structure, for $\mathrm{Ni}_{2} \mathrm{C}$ the structures proposed by Gibson et al. ${ }^{21}$ were used and for $\mathrm{Ni}_{3} \mathrm{C}$ the rhombohedral (bainite, space group 167) structure, which was experimentally found by $\mathrm{Na}$ gakura $^{24}$ was assumed (see figure 1](c)).

All results presented here were obtained applying density functional theory (DFT), in the generalized gradient approximation (GGA) in the Perdew-Burke-Ernzerhof (PBE) parametrization 25 as exchange-correlation functional, which is known to give good results for bulk mechanical properties when comparing to experiments26. The plane-wave implementation in the ABINIT pack$\operatorname{age}^{27}[29]$ was used, employing the projector augmented wave (PAW) method 30 . The PAW atomic data sets treat $3 \mathrm{~d}^{8} 4 \mathrm{~s}^{2}$ and $2 \mathrm{~s}^{2} 2 \mathrm{p}^{2}$ as valence for nickel ${ }^{31}$ and carbon 32 , respectively.

For numerical accuracy, the plane-wave cutoff was converged to $E_{\text {cut }} \sim 980 \mathrm{eV}(=36 \mathrm{Ha})$. Only, for calculations of fcc-nickel, the cutoff was set to about $1360 \mathrm{eV}(50 \mathrm{Ha})$ in order to reach a convergence of total energy below about $2.7 \mathrm{meV}\left(1 \times 10^{-4} \mathrm{Ha}\right)$ per atom. At this point

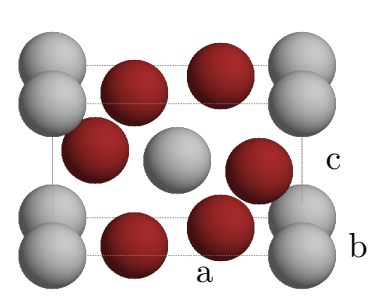

(a) $\mathrm{Ni}_{2} \mathrm{C}(058)$

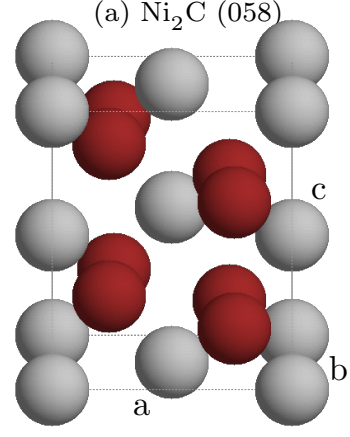

(b) $\mathrm{Ni}_{2} \mathrm{C}(060)$

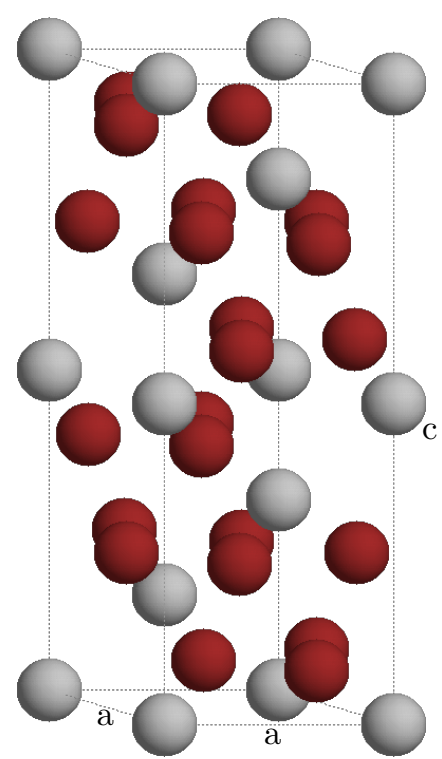

(c) $\mathrm{Ni}_{3} \mathrm{C}(167)$
FIG. 1. (color online) Overview of the crystal structures used in this work for $\mathrm{Ni}_{2} \mathrm{C}$ of space groups 058 (a) two f.u.s and 060 (b) four f.u.s), both orthorhombic, as well as of $\mathrm{Ni}_{3} \mathrm{C}(\mathrm{c})$ space group 167, six f.u.s. The latter is displayed in a hexagonal unit cell for clarity, where the angle enclosed by the $a$ edges in the basal plane is equal to $120^{\circ}$. A primitive rhombohedral cell of only one third the size exists which was used for calculations. The grey balls represent carbon and the red ones represent nickel. The unit vector $\mathbf{e}_{x}$ is always parallel to $a$ and $\mathbf{e}_{z}$ is parallel to $c$. energy differences under strain are converged to below about $0.27 \mu \mathrm{eV}\left(1 \times 10^{-8} \mathrm{Ha}\right)$, which is far more accurate than required for structural relaxation and the calculation of elastic properties. The stronger total energy criterion was chosen with regard to calculating formation enthalpies.

When calculating ground state properties of carbides the Brillouin zone was sampled with a Monkhorst-Pack grid of $12 \times 12 \times 12 k$-points. Thermal smearing of FermiDirac-type ${ }^{33}$ was fixed to about $27 \mathrm{meV}\left(1 \times 10^{-3} \mathrm{Ha}\right)$. Since the unit cells of nickel and diamond are smaller, denser grids of $32 \times 32 \times 32$ and $16 \times 16 \times 16 k$-points, respectively, were required in order to get comparable sampling accuracy.

The ground state formation energies per formula unit (f.u.) for the carbides were calculated according to

$\Delta E_{f}=E_{\mathrm{Ni}_{\mathrm{x}} \mathrm{C}_{\mathrm{y}}}-x E_{\mathrm{fcc}-\mathrm{Ni}}-y\left(E_{\text {diamond }}-25 \mathrm{meV}\right)$

where $E_{i}$ is the total energy of compound $i$. Diamond was calculated as carbon reference structure instead of graphite because the employed method is not capable of correctly calculating van-der-Vaals interactions. An empiric correction of $\Delta E_{\mathrm{C}}=25 \mathrm{meV}$ per carbon atom, also used in reference 21, was applied to obtain formation energies with respect to graphite.

\section{B. Frozen Phonon Calculations}

Within the linear regime, elastic properties can be described by the elastic tensor $\hat{C}$, which gives the stress response $\hat{\sigma}$ of a material proportional to a deformation $\hat{e}$ :

$$
\sigma_{i}=\sum_{j} c_{i j} \cdot e_{j}
$$

Here, Voigt's notation is used to write the stress and deformation tensors as six-vectors $(11 \rightarrow 1 ; 22 \rightarrow 2 ; 33 \rightarrow$ $3 ; 23 \rightarrow 4 ; 13 \rightarrow 5 ; 12 \rightarrow 6$ ), with entries corresponding to three axial strains $(1-3)$ and shear strains $(4-6)$. In this way the elastic tensor can be written as a $6 \times 6$ matrix from which all elastic properties can be derived. The bulk modulus is given by:

$$
B=\frac{1}{3}\left(\left\langle c_{11}\right\rangle+2\left\langle c_{12}\right\rangle\right)
$$

where $\left\langle c_{11}\right\rangle$ denotes an average over the diagonal axial strain entries and $\left\langle c_{12}\right\rangle$ an average over the off-diagonal axial strain entries.

The entries of the elastic tensor were calculated using the frozen phonon (FP) method, where the stressresponse was derived from ground state calculations of the deformed primitive cell. A more detailed explana-

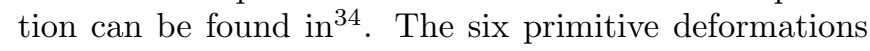
were applied separately with magnitudes ranging up to $1 \%$. All elastic constants were then determined using equation (2). The diagonal entries of the tensor can also 
be determined from the total energies of the same calculations:

$$
E_{\delta}=E_{0}+\frac{V_{0}}{2} \sum_{i} c_{i i} \cdot e_{i}^{2}
$$

where $E_{0}$ and $V_{0}$ are the total energy and volume of the unstrained cell. The calculated tensors were checked for consistency by comparing the results of equations (2) and (4). The calculation parameters were converged until the difference between the diagonal tensor elements from both equations was less than $2 \mathrm{GPa}$. This criterion called for using a $48 \times 48 \times 48 k$-point grid for the deformed cell of $\mathrm{NiC}$, for the other materials it was met by using the aforementioned simulation parameters.

If the material's unit cell exhibits internal degrees of freedom, performing a ground state calculation of the deformed cell without relaxation of the ion positions yields entries of the so-called clamped-ion elastic tensor $\hat{C}^{c}$. To obtain the more physical relaxed-ion elastic tensor $\hat{C}^{r}$, the internal atomic coordinates were relaxed using the Broyden-Fletcher-Goldfarb-Shanno algorithm as implemented in ABINIT until all forces were below $5 \times 10^{-4} \mathrm{eV} / \AA$.

\section{Electronic Transport}

Electronic transport was calculated assuming constant relaxation time $\tau$ within the Boltzmann formalism where the conductivity tensor at zero temperature is given as: $\frac{35}{35}$

$$
\begin{gathered}
\varsigma_{i j}=\tau \frac{e^{2}}{(2 \pi)^{3} \hbar} \sum_{n} \int_{\varepsilon^{n}(\mathbf{k})=E_{\mathrm{Fermi}}} \mathrm{d} S \frac{v_{i}^{n}(\mathbf{k}) v_{j}^{n}(\mathbf{k})}{\left|\mathbf{v}^{n}(\mathbf{k})\right|} \\
\text { with } \quad \mathbf{v}^{n}(\mathbf{k})=\frac{1}{\hbar} \nabla_{\mathbf{k}} \varepsilon^{n}(\mathbf{k})
\end{gathered}
$$

where $\varepsilon^{n}$ is the eigenenergy of the $n$th band and $\mathbf{v}^{n}(\mathbf{k})$ the vector of the corresponding group velocity. $e$ denotes the electron charge and $i, j$ denote cartesian vector components.

Off-diagonal elements of $\varsigma_{i j}$ are zero by symmetry. For the relaxation time $\tau$ no specific value is assumed, though it might be anisotropic $\left(\tau_{z z} \neq \tau_{x x}=\tau_{y y}\right)$ in the case of $\mathrm{Ni}_{3} \mathrm{C}$ due to its rhombohedral structure ${ }^{36}$. The integrals on the right-hand side of equation (5) reflect the anisotropy of the bandstructure of the unperturbed, but eventually strained, systems at the Fermi level. Assuming that $\tau$ remains constant under strain in the linear regime, since no new scattering centers are created, predictions can be made about the change of conductivity under strain.

For strained cells, the bandstructure with relaxed ion positions was used as basis for these calculations.

\section{RESULTS AND DISCUSSION}

\section{A. Ground State Results}

The lattice parameters of the investigated materials are available in literature, some even from experiments. The lattice constant calculated for fcc-nickel in the present work $\left(a_{\mathrm{Ni}}=3.524 \AA\right)$ agrees very well with values found in literature 2137 . The obtained lattice parameter for diamond $\left(a_{\text {diamond }}=3.577 \AA\right)$ is only slightly larger than the experimental value of $3.567 \AA^{38}$. Lattice parameters obtained for the carbides as well as formation enthalpies will be given for comparison, the latter with respect to fcc-Ni and graphite.

a. NiC Assuming rocksalt structure, the lattice parameter $a_{\mathrm{NiC}}=4.073 \AA$ was obtained, which is in good agreement with ref ${ }^{21}(4.077 \AA)$ and other numerical studies cited therein. The calculated formation enthalpy of $\Delta E_{f, \mathrm{NiC}}=49.7 \mathrm{kcal} / \mathrm{mol}$ of f.u. also agrees with ref. ${ }^{21}$ $(48.6 \mathrm{kcal} / \mathrm{mol})$.

TABLE I. Lattice parameters, formation enthalpies and total energies for the two considered orthorhombic structures of $\mathrm{Ni}_{2} \mathrm{C}$. Length values are given in $\AA$, formation enthalpies in $\mathrm{kcal} / \mathrm{mol}$ of f.u. Values from ref. ${ }^{[21}$ are given in parentheses.

\begin{tabular}{lrr}
\hline & $\mathrm{Ni}_{2} \mathrm{C}(058)$ & $\mathrm{Ni}_{2} \mathrm{C}(060)$ \\
\hline$a$ & $4.72(4.72)$ & $4.19(4.19)$ \\
$b$ & $4.19(4.17)$ & $5.51(5.51)$ \\
$c$ & $2.93(2.92)$ & $4.94(4.94)$ \\
$\Delta E_{f}$ & $12.2(7.9)$ & $12.0(7.9)$ \\
\hline
\end{tabular}

b. $\quad \mathrm{Ni}_{2} \mathrm{C}$ The calculated values for the two investigated structures are summarized in table I. The lattice parameters are in excellent agreement with ref.21. Only, the formation enthalpies stated therein disagree with the present results (see table I. values in parentheses). However, there is agreement on the prediction that both structures are essentially degenerate, with the variant of space group 060 being less than $5 \mathrm{meV}$ lower in total energy.

c. $\quad N i_{3} C$ The obtained lattice parameters $a=4.60 \AA$ and $c=13.00 \AA$ are in good agreement with ref ${ }^{21}$ $(a=4.49 \AA, c=13.02 \AA)$ and electron diffraction measurements ${ }^{39}(a=4.553 \AA, c=12.92 \AA)$. A formation enthalpy of $\Delta E_{f, \mathrm{Ni}_{3} \mathrm{C}}=6.3 \mathrm{kcal} / \mathrm{mol}$ was obtained, which is identical to the value reported in ref ${ }^{21}$ and reflects the thermal decomposition observed in 40 .

For the relaxed, strain-free geometries, all carbides of nickel investigated here are meta-stable at $T=0 \mathrm{~K}$, nonmagnetic and metallic as observed previously 21. $\mathrm{Ni}_{2} \mathrm{C}$ exhibits a very low density of states (DOS) around the Fermi energy. The DOS for the investigated carbides and the reference phases are plotted in figure 2 .

In all carbides the $\mathrm{C} 2 \mathrm{~s}$ band is located below the conduction band. It is shifted to lower energies (shifted left in figure 2 for $\mathrm{Ni}_{2} \mathrm{C}$ and $\mathrm{Ni}_{3} \mathrm{C}$ in comparison to the carbide with higher carbon content, $\mathrm{NiC}$, indicating a deeper 


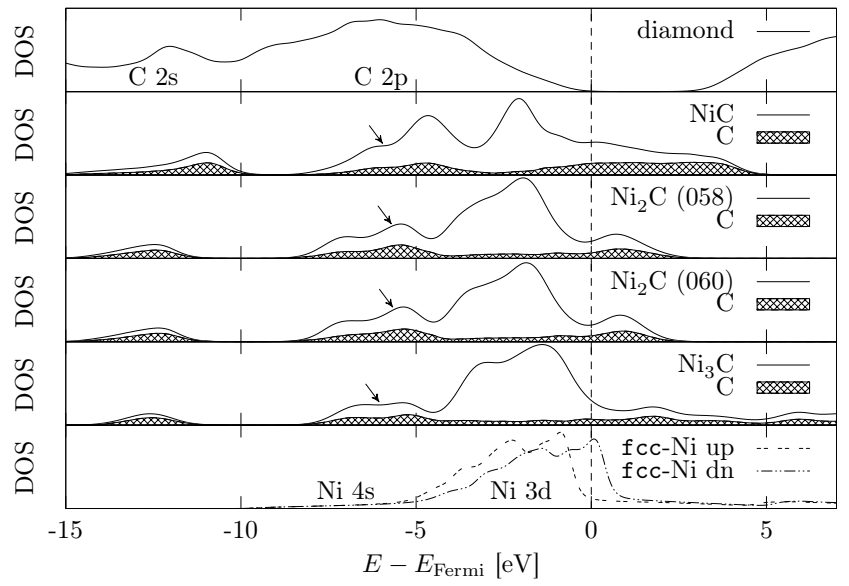

FIG. 2. Total and projected DOS of carbides compared to those of the reference phases. For display, Gaussian smearing of about $270 \mathrm{meV}\left(1 \times 10^{-2} \mathrm{Ha}\right)$ was applied. Atomic orbitals are indicated as obtained from calculations of projected DOS filled areas under curves indicate the fraction of DOS attributed to $\mathrm{C}$ sites. The arrows indicate the bonding band with C $2 p$ and hybrid Ni states.

potential well for electrons is provided by the carbon atoms. They are also more strongly negatively charged than in NiC. The $\mathrm{Ni} 3 \mathrm{~d}$ states are located below the Fermi energy above about $-5 \mathrm{eV}$ for $\mathrm{Ni}_{2} \mathrm{C}$ and $\mathrm{Ni}_{3} \mathrm{C}$, for $\mathrm{NiC}$ they are spread over a broader energy range, starting at around $-6 \mathrm{eV}$. The Ni 3d orbitals do contribute to the DOS at the Fermi level, but much less than in fcc-nickel, where the $3 \mathrm{~d}-\mathrm{DOS}$ of the minority spin peaks at the Fermi level. $\mathrm{Ni} 4 \mathrm{~s}$ and $\mathrm{C} 2 \mathrm{p}$ states also contribute to the DOS at the Fermi level. The part of the conduction band below about $-5 \mathrm{eV}$ is predominately composed of $\mathrm{C} 2 \mathrm{p}$ states hybridizing with $\mathrm{Ni}$ states, see arrows in figure 2 .

\section{B. Elastic Constants}

As a reference, the elastic tensors of $\mathrm{fcc}-\mathrm{Ni}$ and diamond were calculated and the non-zero, not symmetrically equivalent elements are provided in table II. The calculated bulk modulus for diamond is identical to earlier theoretical works ${ }^{41}$ and also the tensor components agree with earlier literature data ${ }^{42}$. The bulk modulus for $\mathrm{Ni}$ is within about $10 \mathrm{GPa}$ of experimental results 43 . This deviation is predominantly attributed to the approximations involved in DFT. The following predictions for the elastic properties of nickel carbides can be expected to have about the same accuracy.

All carbides exhibit a larger bulk modulus than Nickel and a much lower one than diamond, as apparent from the last column of table II. Being the carbide with the highest carbon content, $\mathrm{NiC}$ shows the largest bulk modulus of the carbides. Evidently, the bulk modulus increases with increasing carbon content, that is, the sub- stances become harder. Table II lists all calculated nonzero and not symmetrically equivalent elastic constants. The carbides $\mathrm{Ni}_{2} \mathrm{C}$ and $\mathrm{Ni}_{3} \mathrm{C}$ exhibit less symmetric unit cells, resulting in more independent entries in the elastic tensor.

d. $\quad \mathrm{Ni}_{2} \mathrm{C}$ Both investigated hypothetical forms of $\mathrm{Ni}_{2} \mathrm{C}$ are predicted to be equally hard and even show quite similar anisotropies, probably due to the fact that both are orthorhombic. The elastic properties of the sample should not depend on the relative prevalence of these two phases. Still, judging by the elastic tensors, deforming one cell into the equilibrium shape of the other and allowing the atoms to rearrange into the other structure by relaxation requires overcoming a large potential barrier. Thus, even under stress, both structures can be expected to coexist in one sample.

e. $\quad \mathrm{Ni}_{3} \mathrm{C}$ Judging by the obtained bulk moduli, a macroscopically isotropic polycrystalline sample of $\mathrm{Ni}_{3} \mathrm{C}$ is predicted to be about as hard as the less stable $\mathrm{Ni}_{2} \mathrm{C}$. Even for the most extreme simulated deformations of $1 \%$ stresses were found to be still in the linear regime. Using the calculated value for $c_{11}^{r}$, a compression in $\mathbf{e}_{1}$-direction of this magnitude corresponds to applying a pressure of about $2.7 \mathrm{GPa}$, which by far exceeds pressures achievable in most experiments.

Investigating $\mathrm{DOS}$ and band structure of deformed cells, no qualitative difference with respect to that obtained for the equilibrium geometry was found. For purely axial strains and compressions (i.e. $\mathbf{e}_{1}, \mathbf{e}_{2}$ and $\mathbf{e}_{3}$, see figure $3(\mathrm{~b})$ bands move slightly closer to the Fermi-level under strain and further away under compression. This can be attributed to changing overlaps between atomic orbitals. This difference is marginal close to and at the Fermi level, the region most relevant to transport properties. For pure shear deformations no significant changes are observed, see figure $3(\mathrm{c})$. Band structures shown in figure 3 for deformed cells use relaxed ion positions. Clamped cells show qualitatively identical changes, only the shift of bands for strains and compressions is larger.

\section{Electronic Transport under Strain}

A closer analysis of the $\mathrm{Ni}_{3} \mathrm{C}$ band structure yields a density of states of $2.83 \mathrm{eV}^{-1}$ per f.u. and anisotropic averaged Fermi velocities of $v_{x}=v_{y}=0.90 \times 10^{6} \mathrm{~m} / \mathrm{s}$ and $v_{z}=1.10 \times 10^{6} \mathrm{~m} / \mathrm{s}$. This results in an in-plane/outof-plane conductivity anisotropy of about 0.67 .

The respective effect of axial strains in $x$ and $z$ direction $\left(\mathbf{e}_{1}\right.$ and $\left.\mathbf{e}_{3}\right)$ on electronic transport was investigated. For strains up to $\pm 1 \%$ the DOS remains unaffected within the precision of the calculation. Table III summarizes relative changes under strain in both conductance and Fermi velocities. The strongest changes in conductance can be observed for strains along $\mathbf{e}_{3}$, where the parallel conductance in $z$ direction changes by $10 \%$, the anisotropy increases slightly. For small strains along 
TABLE II. Calculated elastic constants, $c_{i j}$ and bulk modulus $B$, for the considered carbide phases and the reference phases diamond and fcc-Nickel in GPa. For phases with atomic degrees of freedom on the unit cell $\left(\mathrm{Ni}_{2} \mathrm{C}, \mathrm{Ni}_{3} \mathrm{C}\right)$ both clamped-ion and relaxed-ion results are listed. All omitted entries are given by or are zero by symmetry. For space group $167\left(\mathrm{Ni}_{3} \mathrm{C}\right)$ the following relation holds: $c_{56}=c_{14}=-c_{24}$.

\begin{tabular}{|c|c|c|c|c|c|c|c|c|c|c|c|}
\hline & $c_{11}$ & $c_{22}$ & $c_{33}$ & $c_{12}$ & $c_{13}$ & $c_{23}$ & $c_{44}$ & $c_{55}$ & $c_{66}$ & $c_{14}$ & $\bar{B}$ \\
\hline diamond & 1049 & & & 129 & & & 564 & & & & 435 \\
\hline $\mathrm{NiC}$ & 296 & & & 231 & & & 50 & & & & 256 \\
\hline $\mathrm{Ni}_{2} \mathrm{C}(058)$, & 316 & 262 & 378 & 218 & 175 & 193 & 116 & 91 & 145 & & 236 \\
\hline $\mathrm{Ni}_{2} \mathrm{C}(058)$, & 307 & 234 & 344 & 203 & 160 & 163 & 88 & 87 & 145 & & 215 \\
\hline $\mathrm{Ni}_{2} \mathrm{C}(060)$ & 279 & 343 & 359 & 213 & 203 & 171 & 90 & 125 & 135 & & 239 \\
\hline $\mathrm{Ni}_{2} \mathrm{C}(060)$, relaxed & 251 & 333 & 335 & 205 & 186 & 163 & 78 & 91 & 113 & & 225 \\
\hline $\mathrm{Ni}_{3} \mathrm{C}$ clamped & 321 & & 309 & 176 & 184 & & 116 & & 72 & -11 & 227 \\
\hline $\mathrm{Ni}_{3} \mathrm{C} \mathrm{re}$ & 272 & & 276 & 157 & 150 & & 91 & & 57 & -22 & 219 \\
\hline $\mathrm{fcc}-\mathrm{Ni}$ & 266 & & & 156 & & & 129 & & & & 192 \\
\hline
\end{tabular}

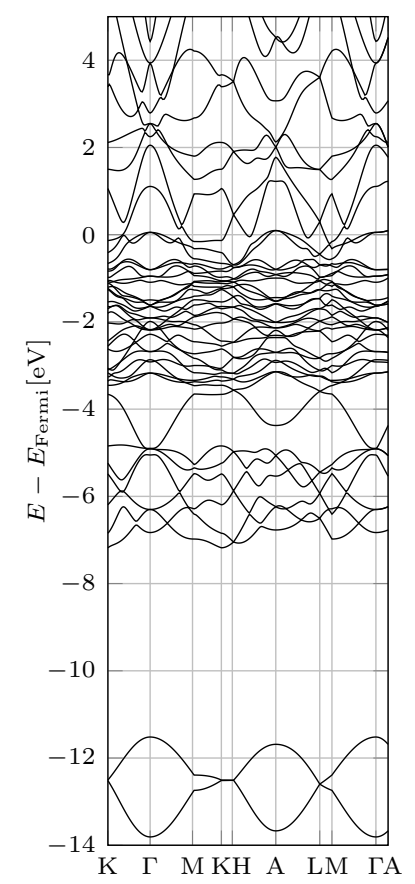

(a) equilibrium

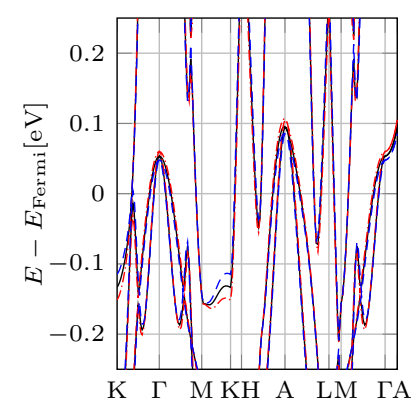

(b) $e_{3}$ (strain)

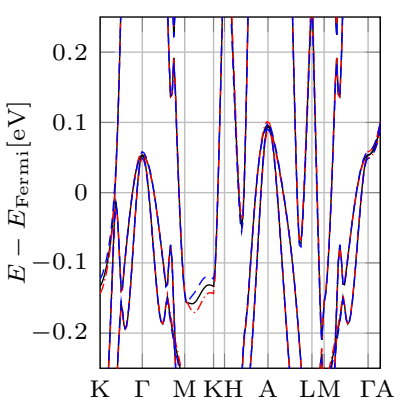

(c) $e_{4}$ (shear)
FIG. 3. (color online) Band structure of equilibrium $\mathrm{Ni}_{3} \mathrm{C}$ for a large energy range (a) and for a small interval around $E_{\mathrm{Fermi}}$ in comparison with $1 \%$ uniaxially distorted cells (b) and (c)]. The displayed deformation directions are the ones corresponding to the largest strain (b) and shear (c) components of the relaxed elastic tensor. Bands of positively deformed cells are plotted in red dash-dotted lines, negative deformations in blue dashed lines.

$\mathbf{e}_{1}$ the in-plane isotropy $(x x, y y)$ is unaffected, while the in-plane/out-of-plane anisotropy changes slightly.

Computational and analytical investigations of the piezoresistivity of semi-conducting CNTs suggest an increase in resistance of well above $50 \%$ under longitudinal strains of about $0.3 \% 445$. To exert the required stress on $\mathrm{Ni}_{3} \mathrm{C}$-contacted $\mathrm{CNT}$ the contact material would be strained by about $1 \%$, given the ratio of the established Young's modulus of CNTs of about $1000 \mathrm{GPa}^{46}$ and the elastic coefficients we obtained for axial strains in $\mathrm{Ni}_{3} \mathrm{C}$.
This would cause a change in conductivity of 3 to $10 \%$ in the contact material, which is significantly smaller than the effect observed in the CNT. Thus, the piezoresistive properties of a device with Ni-contacted CNTs as functional structure are dominated by the electronic response of the CNTs to mechanical deformations.

For isotropic compression of a polycrystalline sample, linear combination of the axial effects suggests a reduction of conductivity. This is mostly a result of the relatively large decrease in conductivity by axial compression in $z$ direction.

The piezoresistive effect observed by Uhlig et al. in $\mathrm{Ni}_{3} \mathrm{C}$-containing nickel-carbon thin films ${ }^{13}$ under hydrostatic pressure is opposite to this prediction for bulk $\mathrm{Ni}_{3} \mathrm{C}$ based on our calculations. Thus our study excludes the possibility, that these observations are dominated by bulk effects in $\mathrm{Ni}_{3} \mathrm{C}$ grains. One may speculate that they emerge from effects in nickel grains, since nickel itself is known to show piezoresistive effects 4748 . Effects at interfaces in the nickel-carbon mixture may also play a role.

TABLE III. Relative changes in transport properties of $\mathrm{Ni}_{3} \mathrm{C}$ under strain in $\mathbf{e}_{1}(x)$ and $\mathbf{e}_{3}(z)$ direction, respectively. Within the accuracy of the calculations, transport coefficients in $x$ and $y$ directions are affected equally by the considered strain values.

\begin{tabular}{lrrrr}
\hline strain & $\Delta \varsigma_{x x} / \varsigma_{x x}$ & $\Delta \varsigma_{z z} / \varsigma_{z z}$ & $\Delta v_{x} / v_{x}$ & $\Delta v_{z} / v_{z}$ \\
\hline$e_{1}=+1 \%$ & $-3 \%$ & 0 & $-2 \%$ & $-0.4 \%$ \\
$e_{1}=-1 \%$ & $+3 \%$ & 0 & $+2 \%$ & $+0.6 \%$ \\
$e_{3}=+1 \%$ & $+4 \%$ & $+10 \%$ & $+1 \%$ & $+4 \%$ \\
\hline
\end{tabular}

\section{CONCLUSIONS}

The complete sets of elastic constants of nickel carbides have been calculated in a way that they can be expected to be within $10 \mathrm{GPa}$ of experimental values. The electronic structure and electronic transport properties of bulk $\mathrm{Ni}_{3} \mathrm{C}$ under stress have been investigated. Assuming a constant relaxation time $\tau$, changes in conductivity not exceeding about $4 \%$ in-plane and about $10 \%$ out-of-plane for stresses below $3 \mathrm{GPa}$ are predicted. As 
a contact material in sensing applications these changes are of minor significance. These results also show, that $\mathrm{Ni}_{3} \mathrm{C}$ does not contribute significantly to the piezoresistive effects observed in nickel-carbon thin films by Uhlig and coworkers ${ }^{1}$.

For the formation enthalpy of both $\mathrm{Ni}_{2} \mathrm{C}$ variants, the absolute values obtained here differ quantitatively from those by Gibson et al ${ }^{21}$, but qualitatively both studies agree on the relative ordering with respect to the other carbide phases. On the enthalpy difference between the ground state $\mathrm{Ni}_{2} \mathrm{C}$ variants the agreement is excellent. The studies also agree on the formation enthalpies of the other carbides.

\section{ACKNOWLEDGMENTS}

We thank R. Wenisch for fruitful discussions. This work has been partially financed by the Initiative and
Networking Fund of the German Helmholtz Association via the Helmholtz International Research School NanoNet (VH-KO-606) and the W2/W3 Programm für exzellente Wissenschaftlerinnen (W2/W3-026). We gratefully acknowledge partial funding by the DFG via Research Unit FOR1713 (SMINT) and the Center for Advancing Electronics Dresden (cfaed). We thank the HZDR computing center for provided computational resources.
* j.kelling@hzdr.de

†.gemming@hzdr.de

1 Steffen Uhlig, Hanna Schmid-Engel, Tobias Speicher, and Günter Schultes. Pressure sensitivity of piezoresistive nickel-carbon Ni:a-C:H thin films. Sens. Actuators A, 193(0):129-135, 2013. ISSN 0924-4247. doi:10.1016/j.sna.2012.12.027. URL http://www.sciencedirect.com/science/article/ pii/S0924424712007649

2 Zachary L Schaefer, Kaitlyn M Weeber, Rajiv Misra, Peter Schiffer, and Raymond E Schaak. Bridging hcp-Ni and Ni3C via a Ni3C1-x Solid Solution: Tunable Composition and Magnetism in Colloidal Nickel Carbide Nanoparticles. Chem. Mater., 23(9):2475-2480, 2011.

3 Steffen Uhlig, Rudolf Struis, Hanna Schmid-Engel, Jochen Bock, Anne-Catherine Probst, Olivia Freitag-Weber, Ivo Zizak, Roman Chernikov, and Günter Schultes. Piezoresistive Ni:a-C:H thin films containing hcp-Ni or Ni3C investigated by XRD, EXAFS, and wavelet analysis. Diam. Relat. Mater., 34(0):25-35, 2013. ISSN 0925-9635. doi:10.1016/j.diamond.2013.01.013 URL http://www.sciencedirect.com/science/article/pii/ S092596351300023X.

${ }^{4}$ Lin He. Hexagonal close-packed nickel or Ni3C? Journal of Magnetism and Magnetic Materials, 322(14):1991-1993, 2010. ISSN 0304-8853. doi:10.1016/j.jmmm.2010.01.020 URL http://www.sciencedirect.com/science/ article/pii/S0304885310000387

Andrej Furlan, Jun Lu, Lars Hultman, Ulf Jansson, and Martin Magnuson. Crystallization characteristics and chemical bonding properties of nickel carbide thin film nanocomposites. J. Phys. Condens. Matter, 26(41):415501, 2014. URL http://stacks.iop.org/0953-8984/26/i= $41 / a=415501$

${ }^{6}$ Bernhard C. Bayer, David A. Bosworth, F. Benjamin Michaelis, Raoul Blume, Gerlinde Habler, Rainer Abart, Robert S. Weatherup, Piran R. Kidambi, Jeremy J. Baumberg, Axel Knop-Gericke, Robert Schloegl, Carsten Bae- htz, Zoe H. Barber, Jannik C. Meyer, and Stephan Hofmann. In Situ Observations of Phase Transitions in Metastable Nickel (Carbide)/Carbon Nanocomposites. J. Phys. Chem. C, 120(39):22571-22584, 2016. doi: 10.1021/acs.jpcc.6b01555 URL http://dx.doi.org/10. 1021/acs.jpcc.6b01555.

Shintaro Sato, Akio Kawabata, Mizuhisa Nihei, and Yuji Awano. Growth of diameter-controlled carbon nanotubes using monodisperse nickel nanoparticles obtained with a differential mobility analyzer Chem. Phys. Lett., 382(3-4):361-366, 2003. ISSN 0009-2614. doi:10.1016/j.cplett.2003.10.076 URL http://www.sciencedirect.com/science/article/pii/ S0009261403018608

8 Anders Börjesson and Kim Bolton. First Principles Studies of the Effect of Nickel Carbide Catalyst Composition on Carbon Nanotube Growth. J. Phys. Chem. C, 114(42): 18045-18050, 2010. doi:10.1021/jp1045707. URL http: //pubs.acs.org/doi/abs/10.1021/jp1045707

y Matthias Krause, Miro Haluška, Gintautas Abrasonis, and Sibylle Gemming. SWCNT growth from C:Ni nanocomposites. Phys. Status Solidi B, 249(12):2357-2360, 2012. ISSN 1521-3951. doi:10.1002/pssb.201200107. URL http: //dx.doi.org/10.1002/pssb.201200107

10 Ming Lin, Joyce Pei Ying Tan, Chris Boothroyd, Kian Ping Loh, Eng Soon Tok, and Yong-Lim Foo. Direct Observation of Single-Walled Carbon Nanotube Growth at the Atomistic Scale. Nano Lett., 6(3):449-452, 2006. doi: 10.1021/nl052356k. URL http://dx.doi.org/10.1021/ $\mathrm{nl052356 \textrm {k }}$.

11 C. Ducati, I. Alexandrou, M. Chhowalla, J. Robertson, and G. A. J. Amaratunga. The role of the catalytic particle in the growth of carbon nanotubes by plasma enhanced chemical vapor deposition. J. Appl. Phys., 95(11):6387-6391, 2004. doi: 10.1063/1.1728293 URL http://scitation.aip.org/ content/aip/journal/jap/95/11/10.1063/1.1728293. 
12 Wei Zhou, Kun Zheng, Lin He, Rongming Wang, Lin Guo, Chinping Chen, Xiaodong Han, and Ze Zhang. Ni/Ni3C Core-Shell Nanochains and Its Magnetic Properties: OneStep Synthesis at Low Temperature. Nano Lett., 8(4): 1147-1152, 2008. doi:10.1021/nl073291j. URL http: //dx.doi.org/10.1021/n1073291j. PMID: 18358008.

${ }^{13}$ Qing Cao, Shu-Jen Han, Jerry Tersoff, Aaron D. Franklin, Yu Zhu, Zhen Zhang, George S. Tulevski, Jianshi Tang, and Wilfried Haensch. End-bonded contacts for carbon nanotube transistors with low, size-independent resistance. Science, 350(6256):68-72, 2015. ISSN 00368075. doi:10.1126/science.aac8006. URL http://science. sciencemag.org/content/350/6256/68.

14 James R. McDonough, Jang Wook Choi, Yuan Yang, Fabio La Mantia, Yuegang Zhang, and Yi Cui. Carbon nanofiber supercapacitors with large areal capacitances. Appl. Phys. Lett., 95(24):243109, 2009. doi: 10.1063/1.3273864. URL http://scitation.aip.org/ content/aip/journal/apl/95/24/10.1063/1.3273864

15 Maxwell Zheng, Kuniharu Takei, Benjamin Hsia, Hui Fang, Xiaobo Zhang, Nicola Ferralis, Hyunhyub Ko, YuLun Chueh, Yuegang Zhang, Roya Maboudian, and Ali Javey. Metal-catalyzed crystallization of amorphous carbon to graphene. Appl. Phys. Lett., 96(6):063110, 2010. doi:10.1063/1.3318263. URL http://scitation.aip.org/ content/aip/journal/apl/96/6/10.1063/1.3318263.

to R. Wenisch, R. Hübner, F. Munnik, S. Melkhanova, S. Gemming, G. Abrasonis, and M. Krause. Nickelenhanced graphitic ordering of carbon ad-atoms during physical vapor deposition. Carbon, 100:656-663, 2016. ISSN 0008-6223. doi:10.1016/j.carbon.2015.12.085 URL http://www.sciencedirect.com/science/article/pii/ S0008622315305510.

17 Samuel Grandthyll, Stefan Gsell, Michael Weinl, Matthias Schreck, Stefan Hüfner, and Frank Müller. Epitaxial growth of graphene on transition metal surfaces: chemical vapor deposition versus liquid phase deposition. $J$. Phys. Condens. Matter, 24(31):314204, 2012. URL http: //stacks. iop.org/0953-8984/24/i=31/a=314204

18 Robert S. Weatherup, Bernhard C. Bayer, Raoul Blume, Caterina Ducati, Carsten Baehtz, Robert Schlögl, and Stephan Hofmann. In Situ Characterization of Alloy Catalysts for Low-Temperature Graphene Growth. Nano Lett., 11(10):4154-4160, 2011. doi:10.1021/nl202036y URL http://dx.doi.org/10.1021/nl202036y

19 Jayeeta Lahiri, Travis S Miller, Andrew J Ross, Lyudmyla Adamska, Ivan I Oleynik, and Matthias Batzill. Graphene growth and stability at nickel surfaces. New J. Phys., 13(2):025001, 2011. URL http://stacks .iop.org/ $1367-2630 / 13 / i=2 / a=025001$.

20 Peter Jacobson, Bernhard Stöger, Andreas Garhofer, Gareth S. Parkinson, Michael Schmid, Roman Caudillo, Florian Mittendorfer, Josef Redinger, and Ulrike Diebold. Nickel Carbide as a Source of Grain Rotation in Epitaxial Graphene. ACS Nano, 6(4):3564-3572, 2012. doi: 10.1021/nn300625y. URL http://dx.doi.org/10.1021/ $\mathrm{nn} 300625 \mathrm{y}$

${ }^{21}$ Josh S Gibson, Jamal Uddin, Thomas R Cundari, Nelli K Bodiford, and Angela K Wilson. First-principle study of structure and stability of nickel carbides. J. Phys. Condens. Matter, 22(44):445503, 2010. URL http://stacks. iop.org/0953-8984/22/i=44/a=445503.

${ }^{22}$ Christian Wagner, Jörg Schuster, and Thomas Gessner. DFT investigations of the piezoresistive effect of car- bon nanotubes for sensor application. Phys. Status Solidi B, 249(12):2450-2453, 2012. ISSN 1521-3951. doi: 10.1002/pssb.201200113. URL http://dx.doi.org/10. 1002/pssb. 201200113.

23 A. Erbe, W. Jiang, Z. Bao, D. Abusch-Magder, D. M. Tennant, E. Garfunkel, and N. Zhitenev. Nanoscale patterning in application to materials and device structures. J. Vac. Sci. Technol. B, 23(6):3132-3137, 2005. doi:10.1116/1.2130353 URL http://scitation.aip.org/ content/avs/journal/jvstb/23/6/10.1116/1.2130353.

${ }^{24}$ Sigemaro Nagakura. Study of Metallic Carbides by Electron Diffraction Part I. Formation and Decomposition of Nickel Carbide. J. Phys. Soc. Jpn., 12(5):482-494, 1957. doi:10.1143/JPSJ.12.482. URL http://jpsj.ipap. jp/link?JPSJ/12/482

25 John P. Perdew, Kieron Burke, and Matthias Ernzerhof. Generalized Gradient Approximation Made Simple. Phys. Rev. Lett., 77(18):3865-3868, 1996. doi:10.1103/PhysRevLett.77.3865. URL http://link.aps.org/doi/10.1103/PhysRevLett. 77.3865; http://www.bibsonomy.org/bibtex/ 2507b0512ae6a0b380c7ffe99c4ac0648/chengguang

26 Stefan Kurth, John P. Perdew, and Peter Blaha. Molecular and solid-state tests of density functional approximations: LSD, GGAs, and metaGGAs. Int. J. Quant. Chem., 75(4-5):889-909, 1999. ISSN 1097-461X. doi:10.1002/(SICI)1097461X(1999)75:4/5¡889::AID-QUA54¿3.0.CO;2-8. URL http://dx.doi.org/10.1002/(SICI) 1097-461X(1999) 75:4/5<889: :AID-QUA54>3.0.CO

27 Xavier Gonze, B. Amadon, P.-M. Anglade, Jean-Michel Beuken, F. Bottin, P. Boulanger, F. Bruneval, D. Caliste, R Caracas, M. Côté, Thierry Deutsch, Luigi Genovese, Ph. Ghosez, M. Giantomassi, Stefan Goedecker, DR Hamann, P. Hermet, F. Jollet, G. Jomard, and S. Leroux. ABINIT: First-principles approach to material and nanosystem properties. Comput. Phys. Commun., 180(12):2582-2615, 2009. URL http://dblp.uni-trier. $\mathrm{de} / \mathrm{db} /$ journals/cphysics/cphysics180.html\# GonzeAABBBBCCCDGGGGHHJJL09; http://dx.doi.org/ 10.1016/j.cpc.2009.07.007; http://www . bibsonomy . org/bibtex/2ba2264f9584526062c5945ddef54d46f/dblp

28 Xavier Gonze, J. Beuken, R Caracas, F. Detraux, M. Fuchs, G. Rignanese, L. Sindic, M. Verstraete, G. Zerah, F. Jollet, M. Torrent, A. Roy, M. Mikami, P Ghosez, J. Raty, and DC Allan. First-principles computation of material properties: the ABINIT software project. Comput. Mater. Sci., 25(3): 478-492, 2002. doi:10.1016/S0927-0256(02)00325-7. URL http://www.ingentaconnect.com/content/els/ 09270256/2002/00000025/00000003/art00325

29 Xavier Gonze, GM Rignanese, M. Verstraete, JM Beuken, Y Pouillon, R Caracas, F. Jollet, M. Torrent, G Zerah, M. Mikami, P Ghosez, M Veithen, JY Raty, V Olevano, F Bruneval, L Reining, R Godby, G Onida, DR Hamann, and DC Allan. A brief introduction to the ABINIT software package. Z. Kristallogr., 220(5-6):558-562, 2005. ISSN 0044-2968. doi:10.1524/zkri.220.5.558.65066

30 Marc Torrent, François Jollet, François Bottin, Gilles Zérah, and Xavier Gonze. Implementation of the projector augmented-wave method in the ABINIT code: Application to the study of iron under pressure. Comput. Mater. Sci., 42(2):337-351, 2008. ISSN 0927-0256. URL http://dx.doi.org/10.1016/j.commatsci.2007.07.020 
31 ABINIT PAW Atomic Data Ni, September 2014. URL http://www.abinit.org/downloads/PAW2/TABLES/ MAIN/028-ni/Ni-GGA-atompaw

32 Bernd Meyer and Francois Jollet. ABINIT PAW Atomic Data C, September 2014. URL http://www.abinit.org/ downloads/PAW2/TABLES/MAIN/006-c/C-GGA-hard-uspp.

33 ABINIT Input Variable occopt, October 2015. URL http://www .abinit.org/doc/helpfiles/for-v7.10/ input_variables/varbas.html\#occopt.

${ }^{34}$ Martin F.-X. Wagner and Wolfgang Windl. Lattice stability, elastic constants and macroscopic moduli of NiTi martensites from first principles. Acta Mater., 56(20):6232-6245, 2008. ISSN 13596454. doi:10.1016/j.actamat.2008.08.043 URL http://www.sciencedirect.com/science/article/ pii/S1359645408006083

35 J. M. Ziman. Principles of the Theory of Solids. Cambridge University Press, second edition, 1972. ISBN 9781139644075. URL http://dx.doi.org/10.1017/ CB09781139644075. Cambridge Books Online.

${ }^{36}$ B. Yu. Yavorsky, N. F. Hinsche, I. Mertig, and P. Zahn. Electronic structure and transport anisotropy of $\mathrm{Bi}_{2} \mathrm{Te}_{3}$ and $\mathrm{Sb}_{2} \mathrm{Te}_{3}$. Phys. Rev. B, 84:165208, Oct 2011. doi: 10.1103/PhysRevB.84.165208 URL http://link.aps. org/doi/10.1103/PhysRevB.84.165208.

37 A. Hull. A New Method of X-Ray Crystal Analysis. Phys. Rev., 10:661-696, Dec 1917. doi: 10.1103/PhysRev.10.661. URL http://link.aps.org/ doi/10.1103/PhysRev.10.661

38 T. Yamanaka, S. Morimoto, and H. Kanda. Influence of the isotope ratio on the lattice constant of diamond. Phys. Rev. B, 49:9341-9343, Apr 1994. doi: 10.1103/PhysRevB.49.9341. URL http://link.aps.org/ doi/10.1103/PhysRevB.49.9341.

39 Sigemaro Nagakura. Study of Metallic Carbides by Electron Diffraction Part II. Crystal Structure Analysis of Nickel Carbide. J. Phys. Soc. Jpn., 13(9):1005-1014, 1958. doi:10.1143/JPSJ.13.1005. URL http://jpsj.ipap.jp/ link? JPSJ/13/1005.

40 Ray-Tung Chiang, Ray-Kuang Chiang, and Fuh-Sheng Shieu. Emergence of interstitial-atom-free HCP nickel phase during the thermal decomposition of $\mathrm{Ni3C}$ nanoparticles. $R S C A d v ., 4: 19488-19494,2014$. doi: 10.1039/C4RA01874E URL http://dx.doi.org/10.
1039/C4RA01874E

41 Marvin Cohen. Calculation of bulk moduli of diamond and zinc-blende solids. Phys. Rev. B, 32:7988-7991, Dec 1985. doi:10.1103/PhysRevB.32.7988 URL http://link.aps . org/doi/10.1103/PhysRevB.32.7988

${ }^{42}$ R. H. Telling, C. J. Pickard, M. C. Payne, and J. E. Field. Theoretical Strength and Cleavage of Diamond. Phys. Rev. Lett., 84:5160-5163, May 2000. doi: 10.1103/PhysRevLett.84.5160. URL http://link.aps. org/doi/10.1103/PhysRevLett.84.5160

43 S. Rekhi, S.K. Saxena, R. Ahuja, B. Johansson, and J. Hu. Experimental and theoretical investigations on the compressibility of nanocrystalline nickel. J. Mater. Sci., 36(19):4719-4721, 2001. ISSN 0022-2461. doi: 10.1023/A:1017974904559 URL http://dx.doi.org/10. 1023/A\%3A1017974904559.

44 Saša Dmitrović, Ivanka Milošević, Milan Damnjanović, and Tatjana Vuković. Electronic Properties of Strained Carbon Nanotubes: Impact of Induced Deformations. J. Phys. Chem. C, 119(24):13922-13928, 2015. doi: 10.1021/acs.jpcc.5b02455. URL http://dx.doi.org/10. 1021/acs.jpcc.5b02455

45 Christian Wagner, Jörg Schuster, and Thomas Gessner. Empirical transport model of strained CNT transistors used for sensor applications. J. Comput. Electron., 15(3):881-890, 2016. ISSN 1572-8137. doi: 10.1007/s10825-016-0823-4. URL http://dx.doi.org/10. 1007/s10825-016-0823-4

46 Yang Wu, Mingyuan Huang, Feng Wang, X. M. Henry Huang, Sami Rosenblatt, Limin Huang, Hugen Yan, Stephen P. O'Brien, James Hone, and Tony F. Heinz. Determination of the Young's Modulus of Structurally Defined Carbon Nanotubes. Nano Lett., 8(12):4158-4161, 2008. doi:10.1021/nl801563q URL http://dx.doi.org/ $10.1021 / \mathrm{n} 1801563 \mathrm{q}$

$4 r$ G. C. Kuczynski. Effect of Elastic Strain on the Electrical Resistance of Metals. Phys. Rev., 94:61-64, Apr 1954. doi: 10.1103/PhysRev.94.61. URL/http://link.aps.org/doi/ 10.1103/PhysRev.94.61.

${ }^{48}$ E. Klokholm. Piezoresistance in Evaporated Nickel Films. J. Vac. Sci. Technol., 10(1):235-237, 1973. doi: 10.1116/1.1317950 URL http://scitation.aip.org/ content/avs/journal/jvst/10/1/10.1116/1.1317950 